\title{
Correction to: Characteristics of bone metabolism markers during the healing of osteoporotic versus nonosteoporotic metaphyseal long bone fractures: a matched pair analysis
}

\author{
L. Kolios ${ }^{1} \cdot$ M. Hitzler ${ }^{2}$ A. Moghaddam² ${ }^{2}$ C. Takur ${ }^{2} \cdot$ H. Schmidt-Gayk ${ }^{3} \cdot$ B. Höner ${ }^{4} \cdot$ M. Lehnhardt ${ }^{1} \cdot$ P. Grützner ${ }^{2}$. \\ C. Wölff ${ }^{2}$
}

Published online: 20 May 2020

(c) Springer-Verlag GmbH Germany, part of Springer Nature 2020

\section{Correction to: Eur J Trauma Emerg Surg (2012) 38:457-462 https://doi.org/10.1007/s00068-012-0190-1}

The original version of this article unfortunately contained a mistake. The presentation of Table 1 was incorrect. The corrected table (Table 1) is given below.

The original article can be found online at https://doi.org/10.1007/ s00068-012-0190-1.

L. Kolios

leilakolios@freenet.de; lkolios@bgu-ludwigshafen.de

1 Department for Hand, Plastic and Reconstructive Surgery, Burn Care Centre, Clinic for Plastic

Surgery of the University of Heidelberg, BG Trauma

Center Ludwigshafen, Ludwig-Guttmann-Str. 13,

67071 Ludwigshafen, Germany

2 Department for Traumatology and Orthopaedic Surgery, BG Trauma Center Ludwigshafen, Ludwig-Guttmann-Str. 13, 67071 Ludwigshafen, Germany

3 Clinical Laboratory Limbach, Im Breitspiel 15, 69126 Heidelberg, Germany

4 Department of Social and Legal Sciences, SRH University Heidelberg, Ludwig-Guttmann-Str. 6, 69123 Heidelberg, Germany 
Table 1 Table of matched pairs of patients

\begin{tabular}{|c|c|c|c|c|c|c|}
\hline Patient no. & Sex & Age (years) & Fracture localization & Fracture type & Osteosynthesis & Osteoporosis \\
\hline 1 & Female & 57 & Distal radius fracture & A & Plate & Healthy \\
\hline 1 & Female & 59 & Distal radius fracture & A & Plate & Diseased \\
\hline 2 & Female & 62 & Distal radius fracture & A & Plate & Healthy \\
\hline 2 & Female & 61 & Distal radius fracture & A & Plate & Diseased \\
\hline 3 & Female & 57 & Distal radius fracture & A & Plate & Healthy \\
\hline 3 & Female & 57 & Distal radius fracture & $\mathrm{A}$ & Plate & Diseased \\
\hline 4 & Female & 63 & Distal radius fracture & A & Plate & Healthy \\
\hline 4 & Female & 67 & Distal radius fracture & A & Plate & Diseased \\
\hline 5 & Female & 66 & Distal radius fracture & A & Plate & Healthy \\
\hline 5 & Female & 68 & Distal radius fracture & A & Plate & Diseased \\
\hline 6 & Female & 65 & Distal radius fracture & A & Plate & Healthy \\
\hline 6 & Female & 64 & Distal radius fracture & $\mathrm{A}$ & Plate & Diseased \\
\hline 7 & Female & 56 & Distal radius fracture & B & Plate & Healthy \\
\hline 7 & Female & 69 & Distal radius fracture & $\mathrm{B}$ & Plate & Diseased \\
\hline 8 & Female & 60 & Proximal humeral fractures & A & Plate & Healthy \\
\hline 8 & Female & 59 & Proximal humeral fractures & A & Plate & Diseased \\
\hline 9 & Female & 68 & Proximal humeral fractures & $\mathrm{C}$ & Plate & Healthy \\
\hline 9 & Female & 71 & Proximal humeral fractures & $\mathrm{C}$ & Plate & Diseased \\
\hline 10 & Female & 74 & Proximal humeral fractures & $\mathrm{C}$ & Plate & Healthy \\
\hline 10 & Female & 78 & Proximal humeral fractures & $\mathrm{C}$ & Plate & Diseased \\
\hline 11 & Male & 72 & Femoral neck fractures & A & Screws & Healthy \\
\hline 11 & Male & 75 & Femoral neck fractures & A & Screws & Diseased \\
\hline 12 & Male & 64 & Femoral neck fractures & A & DHS & Healthy \\
\hline 12 & Male & 68 & Femoral neck fractures & A & DHS & Diseased \\
\hline 13 & Female & 59 & Intertrochanteric fracture & $\mathrm{B}$ & DHS & Healthy \\
\hline 13 & Female & 63 & Intertrochanteric fracture & $\mathrm{B}$ & DHS & Diseased \\
\hline 14 & Female & 66 & Intertrochanteric fracture & $\mathrm{C}$ & IFN & Healthy \\
\hline 14 & Female & 70 & Intertrochanteric fracture & $\mathrm{C}$ & IFN & Diseased \\
\hline 15 & Male & 64 & Intertrochanteric fracture & B & IFN & Healthy \\
\hline 15 & Male & 68 & Intertrochanteric fracture & B & IFN & Diseased \\
\hline
\end{tabular}

The matching criteria were age ( \pm 5 years), sex (male, female), fracture localization (proximal humerus, distal radius, proximal femur), fracture type (according to AO classification; A-, B-, or C-type fractures), and operation method (plate, intramedullary nail) 